\title{
THE DISTRIBUTION OF THE HILBERT MODULAR TYPE CUSP FORMS OF WEIGHT TWO AND ITS STATISTICAL MODEL
}

\author{
Hirofumi Ishikawa *
}

\begin{abstract}
In this paper we give a distribution of the Hilbert modular type cusp forms over real quadratic number fields, which are closely connected with the Euler number of the Hilbert modular surface, using the trace formula proved by the author. We consider the statistical model for the increasing trend of the dimensions, and analyze their distribution through it.
\end{abstract}

\section{Introduction}

The trace formula of Hecke operators acting on the space of cusp forms of weight two belonging to a Hilbert modular group over a totally real algebraic number field has been given by the author in [Ishikawa(1979)]. In this paper, we will give the distribution of cusp forms of weight two of two variables over real quadratic fields $\mathbf{Q}(\sqrt{D})$ where $D$ is a square-free natural number and $1<D<10000$. As is well known, a cusp form is an eigenfunction of the Laplace-Beltrami operator belonging to the possible minimal discrete spectrum. Also note that the Euler number of the Hilbert modular surface coincides with the dimension of its space plus 4. The dimension is increasing according as multiplying the discriminant. By using the statistical method, we analyze its distribution. The main term of the dimension formula is its volume part. We estimate its error term as the statistical model,

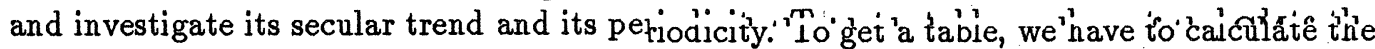
special values of the Dedekind zeta functions and the ldeal class numbers of the vill-nelds with computer's aid. In our table, we can see that the possible minimal eigenvalue of discrete type is missing only when $D=2,3,5,13,17$.

We used ACOS-6 computer system in Okayama University Computer center for about an hour CPU time.

\section{The dimension formula}

For a square-free natural number $D$, let $k$ be a real quadratic field over $\mathbf{Q}$ generated by $\sqrt{D}$. We denote by $\mathrm{g}, E, E^{+}$and $\phi_{i}(i=1,2)$ the ring of integers, the group of units in $\mathrm{g}$, the subgroup of $E$ consisting of all totally positive elements and two distinct projections of $k$ into its completions $\mathbf{R}$ with respect to the infinite valuations. Put $G=\left\{g=\left(g_{1}, g_{2}\right) ; g_{i} \in\right.$ $\left.S L_{2}(\mathbf{R})\right\}, F=\left\{z=\left(z_{1}, z_{2}\right) ; z_{i} \in \mathbf{C}, \operatorname{Re}\left(z_{i}\right) \neq 0\right\}$. We consider that $G$ acts on $F$ by the

\footnotetext{
* College of Liberal Arts and Sciences, Okayama University, Tsushima, Okayama 700, Japan.

Key words : Cusp form; Distribution of dimensions; Hilbert modular; Real quadratic field; Statistical model.
} 


\section{HIROFUMI ISHIKAWA}

linear fractional transformation. We regard $\Gamma=S L_{2}(\mathrm{~g})$ as a subgroup of $G$ through the projections $\phi_{i}$.

By a cusp form of weight two belonging to $\Gamma$, we understand a function $f(z)$ on $F$, satisfying the following conditions:

i) $f(z)$ is holomorphic on each connected component of $F$

ii) $f(\gamma z)=j(\gamma, z)^{-1} f(z)$ for $\gamma \in \Gamma$

where $j(\gamma, z)=\Pi_{i=1,2}\left(c_{i} z_{i}+d_{i}\right)^{-2} \quad\left(\gamma_{i}=\left[\begin{array}{l}a_{i}, b_{i} \\ c_{i}, d_{i}\end{array}\right]\right)$

iii) $f(z)$ vanishes at every parabolic point of $\Gamma$.

The linear space of all $f(z)$ is denoted by $S_{2}(\Gamma)$. The dimension of $S_{2}(\Gamma)$ is given by an eigenvalue of the identity Hecke operator.

Theorem 1. ([Ishikawa(1979), Theorem 1])

$$
\begin{gathered}
\operatorname{dim} S_{2}(\Gamma)=t_{0}+t_{1}+t_{2} \\
t_{0}=\frac{1}{2 \pi^{4}} d_{k}^{3 / 2} \zeta_{k}(2), \quad t_{1}=\sum_{n \geq 1} \frac{4 a_{n}}{n}, \quad t_{2}=-4
\end{gathered}
$$

Here $t_{0}, t_{1}$ and $t_{2}$ are the contributions from identity, elliptic elements and the Eisenstein series. The notations are as follows. $d_{k}, \zeta_{k}$ are the discriminant of $k$, the Dedekind zeta function of $k . a_{n}$ is the number of inequivalent elliptic elements of order $2 n$ in $\Gamma$.

\section{The method of computations}

3.1 By the functional equation of the Dedekind zeta function:

$$
\zeta_{k}(1-s)=\pi^{1-2 s} d_{k}^{s-(1 / 2)} \zeta_{k}(s) \Gamma(s / 2)^{2} / \Gamma((1-s) / 2)^{2},
$$

$t_{0}$ is equal to $2 \zeta_{k}(-1)$. The next lemma is due to [Siegel(1969)].

Lemma 1. For a real quadratic field $k=\mathbf{Q}(\sqrt{D})$ ( $D$ is square-free), we have

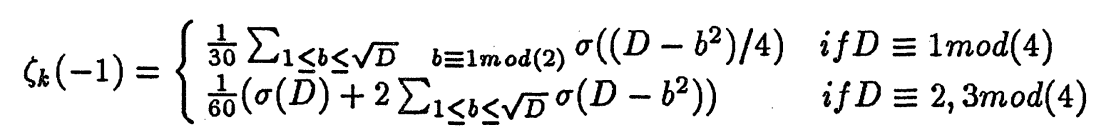

where $\sigma(x)$ is the sum of divisors of $x$.

3.2 Using [Shimizu(1963),No.26], we can rewrite $t_{1}$ as follows.

Lemma 2.

$$
t_{1}=\frac{2}{h_{k}} \sum_{s, \mathbf{f}} \frac{h(\mathbf{o}(s, \mathbf{f}))}{w(\mathbf{o}(s, \mathbf{f}))}(w(\mathbf{o}(s, \mathbf{f}))-1)
$$

Here, $s, \mathbf{f}$ run over integers in $k$, integral ideals in $k$ such that $4-s^{2}$ is totally positive and $\left(s^{2}-4\right) \mathbf{f}^{-2}$ is integral (taken up to $\left.\left(s \pm \sqrt{s^{2}-4}\right) / 2 \bmod (E)\right) . \mathbf{o}=\mathbf{o}(s, \mathbf{f}), h(\mathbf{o})$ and $w(\mathbf{o})$ are the order in $k\left(\sqrt{s^{2}-4}\right)$ with discriminant $\left(s^{2}-4\right) \mathbf{f}^{-2}$, the class number of $\mathbf{o}$ and the index of $E$ in the group of units in $0 . h_{k}$ denotes the class number of $k$. 
The distribution of the Hilbert modular type cusp forms

$s$ is only 0 or \pm 1 except $D=5$, when $s$ satisfies the condition above. For $D=$ $5, s= \pm(1 \pm \sqrt{5}) / 2$ also satisfies it. The possible $\mathbf{f}$ other than $\mathbf{f}=\mathbf{g}$ are $\mathbf{p}, \mathbf{p}^{2}$ for $s=$ $0, D \equiv 3 \bmod (4), \mathbf{p}$ for $s=0, D \equiv 2 \bmod (4)$, and $\mathbf{q}$ for $s= \pm 1, D \equiv 0 \bmod (3)$, where $\mathrm{p}^{2}=2 \mathrm{~g}, \mathrm{q}^{2}=3 \mathrm{~g}$. Then

Lemma 3. When $(s, \mathbf{f})$ satisfies the condition in Lemma 2, we get

$$
\begin{aligned}
& w(\mathbf{o}(0, \mathbf{f}))= \begin{cases}4 & \text { if } D=2, \quad \mathbf{f}=\mathbf{p}, w(\mathbf{o}( \pm 1, \mathbf{f}))=\left\{\begin{array}{ll}
6 & \text { if } D=3, \quad \mathbf{f}=\mathbf{q} \\
2 & \text { otherwise }
\end{array}\right. \text { otherwise }\end{cases} \\
& w(\mathrm{o}( \pm(1 \pm \sqrt{5}) / 2), \mathrm{g}))=5 \quad \text { if } D=5
\end{aligned}
$$

Put $K=k\left(\sqrt{s^{2}-4}\right)$. Let $o_{0}$ be the principal order of $K$. Next we will treat $h(\mathbf{0}) / w(\mathbf{0})$. Considering the numbers of residue classes for the conductor $\mathbf{f}$ of $\mathbf{o}$ in $\mathbf{o}_{0}$, we have

Lemma 4. When $\mathbf{f}$ is the conductor of an order $\mathrm{o}$ in $K$, then

$$
\frac{h(\mathbf{o})}{w(\mathbf{o})}=\frac{h\left(\mathbf{o}_{0}\right)}{w\left(\mathbf{o}_{0}\right)} N(\mathbf{f}) \Pi_{\mathbf{p} \mid \mathbf{f}}\left(1-\left(\frac{K}{\mathbf{p}}\right) N(\mathbf{p})^{-1}\right)
$$

where $(K / \mathbf{p}), N$ stand for the Artin symbol, and for the norm. $\mathbf{p}$ runs over all prime ideals dividing $\mathbf{f}$.

For any case except $s= \pm(1 \pm \sqrt{5}) / 2, K$ is a composite field of $k$ and $\mathbf{Q}(\sqrt{-1})$ or $\mathbf{Q}(\sqrt{-3})$, so $h\left(\mathbf{o}_{0}\right)$ can be expressed by the class numbers of the quadratic fields. The next lemma is given by the consideration of the Dedekind zeta function of $K$.

Lemma 5. For $K=\mathbf{Q}\left(\sqrt{d_{1}}, \sqrt{-d_{2}}\right)\left(d_{1} \geq 2, d_{2} \geq 1\right)$, the ideal class number of $K$ is given by

$$
h\left(\mathbf{o}_{0}\right)=\delta_{2}(1 / 2) h\left(d_{1}\right) h\left(-d_{2}\right) h\left(-d_{1} d_{2}\right)
$$

where $h(d)$ denotes the class number of $\mathbf{Q}(\sqrt{d}) . \delta_{2}=2$ or 1 , according to $\left(d_{1}, d_{2}\right)=$ $(2,1),(2,2)$ or not.

On the other hand, by the direct calculation, we get

$$
h(\mathrm{o}( \pm(1 \pm \sqrt{5}) / 2, \mathrm{~g}))=1
$$

Summing up above things, we obtain

\section{Theorem 2.}

$$
t_{1}=a(D) h(-D)+b(D) h(-3 D)+c(D)
$$

$a(D), b(D)$ and $c(D)$ are given in the following tables. 


\section{HIROFUMI ISHIKAWA}

\begin{tabular}{cccccccc}
\hline$D$ & $D \equiv 1 \bmod (4)$ & $\begin{array}{c}D \equiv 2 \bmod (4) \\
D \neq 2\end{array}$ & $\begin{array}{c}D \equiv 3 \bmod (8) \\
D \neq 3\end{array}$ & $D \equiv 7 \bmod (8)$ & $D=2$ & $D=3$ \\
\hline $2 a(D)$ & 1 & 3 & 10 & 4 & 5 & 3 \\
\hline \multicolumn{7}{c}{} \\
\hline$D$ & $D \equiv 1,2 \bmod (3)$ & $\begin{array}{c}D \equiv 3 \bmod (9) \\
D \neq 3\end{array}$ & $D \equiv 6 \bmod (9)$ & $D=3$ & $D$ & $D=5$ & $D \neq 5$ \\
\hline $6 b(D)$ & 4 & 20 & 12 & 13 & $5 c(D)$ & 8 & 0 \\
\hline
\end{tabular}

\section{The statistical model}

We can calculate the dimension $S_{2}(\Gamma)$ with the aid of Theorem 2. In this section, we regard the dimension as a function of discriminant $x$ of a real quadratic field, and consider the relation $\left(x, \operatorname{dim} S_{2}(x)\right)$ statistically.

We have computed $\operatorname{dim} S_{2}(x)$ for $x$ below 10000 using the computer, and put up the correlation diagram of $\left(x, \operatorname{dim} S_{2}(x)\right)$ in Figure 1.

From the arguments in $\S \S 2-3$, its dimension consists of the volume part and the elliptic part, which are referred to the special value of the Dedekind zeta function and the sum of class numbers of the imaginary quadratic fields, respectively. We estimate the volume part as

$$
\begin{aligned}
& \frac{1}{2 \pi^{4}} x^{3 / 2} \zeta_{k}(2)=\frac{1}{2 \pi^{4}} x^{3 / 2} \zeta_{\mathbf{Q}}(2) \Pi_{p}\left(1-(x / p) p^{-2}\right)^{-1} \\
\sim & \frac{1}{2 \pi^{4}} x^{3 / 2} \zeta_{\mathbf{Q}}(2) \Pi_{p}\left(1+p^{-2}\right)^{-1}=\frac{1}{180} x^{3 / 2} \quad(x \rightarrow \infty)
\end{aligned}
$$

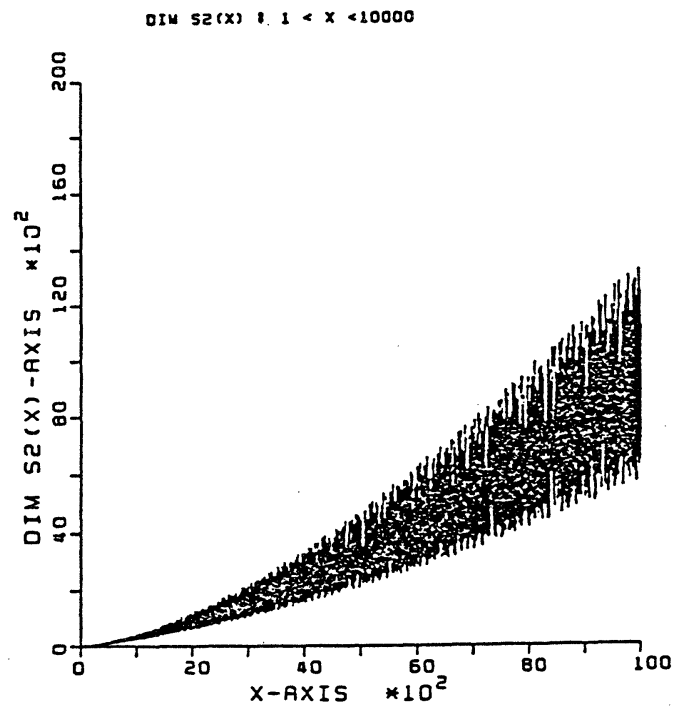

Fig. 1
$\operatorname{LOG}(E(x)), 1<x<10000$

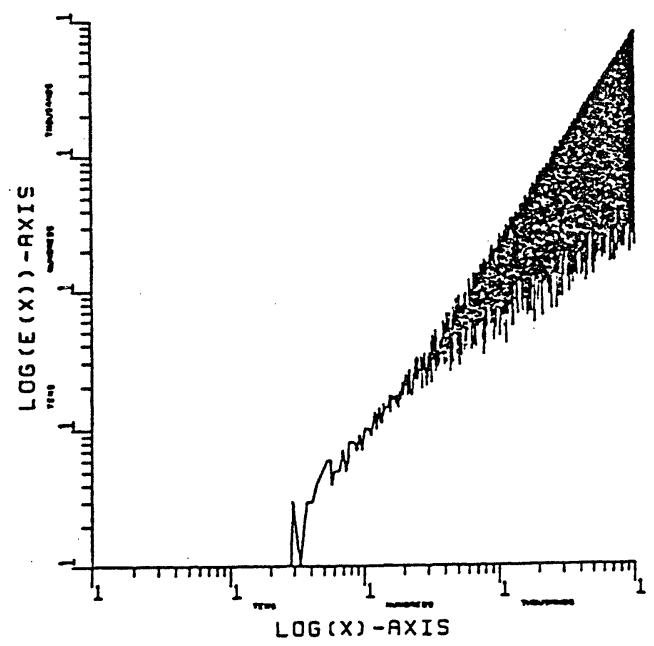

Fig. 2 
where $\zeta_{\mathbf{Q}}$ denotes the Riemann zeta function. Comparing above with the estimates of the class numbers of the imaginary quadratic fields, in the asymptotic expansion, the main term of $\operatorname{dim} S_{2}(x)$ becomes to $(1 / 180) x^{3 / 2}$. We define an error term by.

$$
\varepsilon(x)=\operatorname{dim} S_{2}(x)-(1 / 180) x^{3 / 2}
$$

Now we deal with the error term $\varepsilon(x)$. We give the correlation diagram of $(\log (x)$, $\log \varepsilon(x))$ in Figure 2. Moreover we get the interesting results that the coefficient of correlation between $(\log (x), \log \varepsilon(x))$ is $0.8801(1<x<10000)$. To get its secular trend, we treat the data with the moving average method. In figure 3 , we show a graph of the error variance with the data processed by the moving average method of step below 20. It is decreasing rapidly on $[5,8]$, and slowly on $[9,16]$. As in Figure 4, we present the correlation diagram of the data with the moving average method of step 10, whose error variance is lower than 0.1 .

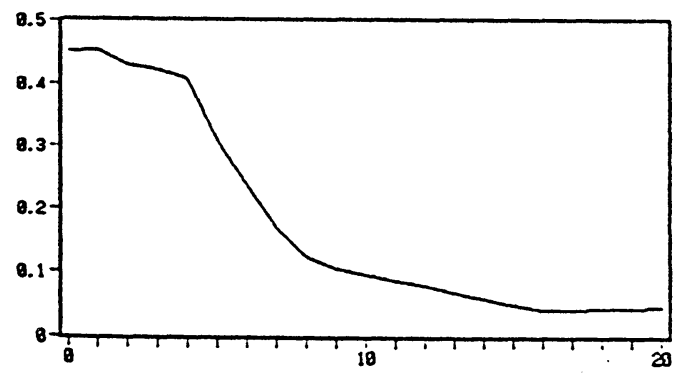

Fig. 3

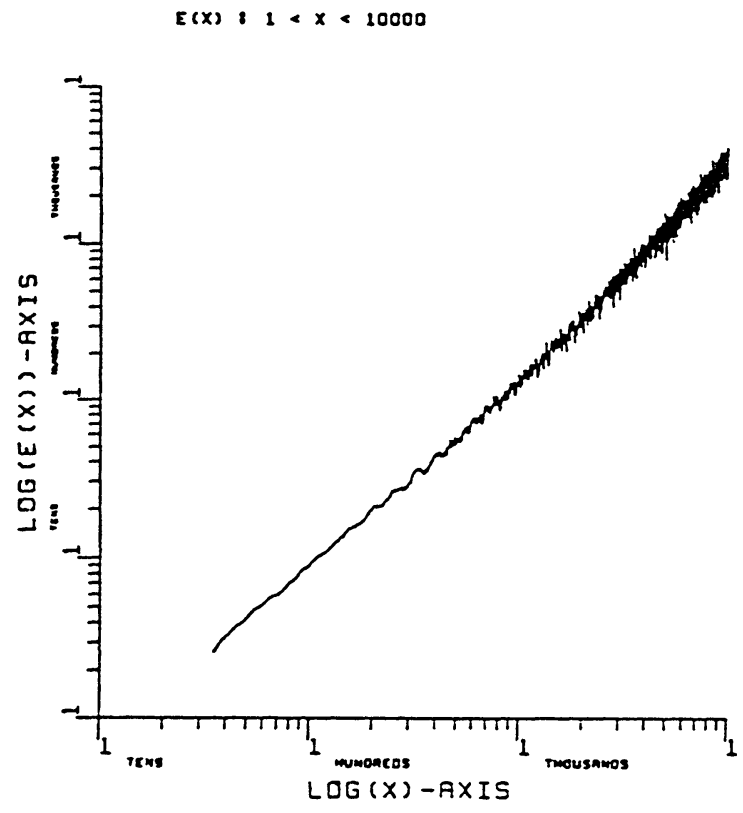

Fig. 4

Following from Fig. 4, its secular trend increases almost straight in this interval. Therefore we consider the following statistical model for this error term.

$$
\varepsilon(x)=\alpha x^{\beta}
$$

By means of the regression analysis, we estimate $(\alpha, \beta)$ for a sample space of $(\log (x)$, $\log \varepsilon(x))(1<x<10000)$. The exponent $\beta$ is more important than $\alpha$ in our case. The 


\section{HIROFUMI ISHIKAWA}

estimates of $(\alpha, \beta)$ are given in the following table.

\begin{tabular}{|c|c|c|c|}
\hline interval & $\alpha$ & $\beta$ & error variance \\
\hline $1 \sim 9999$ & 0.01670 & 1.295 & 0.452 \\
\hline $1 \sim 999$ & 0.04180 & 1.152 & 0.105 \\
$1001 \sim 1999$ & 0.01683 & 1.284 & 0.280 \\
$2001 \sim 2999$ & 0.00974 & 1.356 & 0.383 \\
$3001 \sim 3999$ & 0.00762 & 1.385 & 0.439 \\
$4001 \sim 4999$ & 0.02122 & 1.264 & 0.493 \\
$5001 \sim 5999$ & 0.01162 & 1.336 & 0.514 \\
$6001 \sim 6999$ & 0.01408 & 1.316 & 0.539 \\
$7001 \sim 7999$ & 0.04205 & 1.195 & 0.581 \\
$8001 \sim 8999$ & 0.00395 & 1.461 & 0.554 \\
$9001 \sim 9999$ & 0.00654 & 1.404 & 0.582 \\
\hline
\end{tabular}

The table above says that $\beta$ increases slightly in this interval. $\beta$ is approximately equal to 1.295 , say $\beta_{0}$.

Next we study the cyclical variation of $\varepsilon(x)$. Put

$$
\eta(x)=\log \varepsilon(x)-\beta_{0} \log (x)-\log \alpha_{0} \quad\left(\alpha_{0}=0.01670\right)
$$

We carry out the auto-correlation analysis and the spectral analysis for the data $\{\eta(x) ; 1<$ $x<10000\}$. We show its correlogram, its power spectrum in Figure 5, 6. There exists $\eta(x)$ only when $x$ is a discriminant of real quadratic field; the characteristic function has a period of 4 . The data restricted to $x \equiv 1 \bmod (4)$ account for three quarter of all the data. It follows from (11) that, as to the volume part, the estimate of $t_{0}$ for $x \equiv 1 \bmod (8)$ and that for $x \equiv 5 \bmod (8)$ are in the ratio $5: 3$. Therefore we expect there is a period of 8 , which appears in Fig. 6.
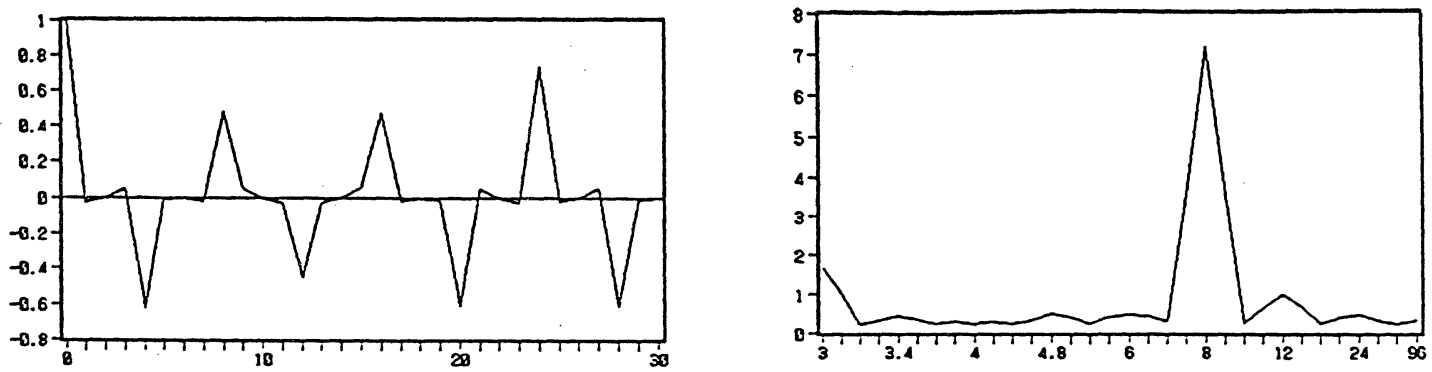

Fig. 5

Fig. 6

To examine its structure minutely, we give the correlogram and the power spectrum for the data $\{\eta(x) ; x \equiv 1 \bmod (8)\}$ in Figure 7,8 . For the case of $x \equiv 5 \bmod (8), x \equiv 8 \bmod (16)$ or 
The distribution of the Hilbert modular type cusp forms

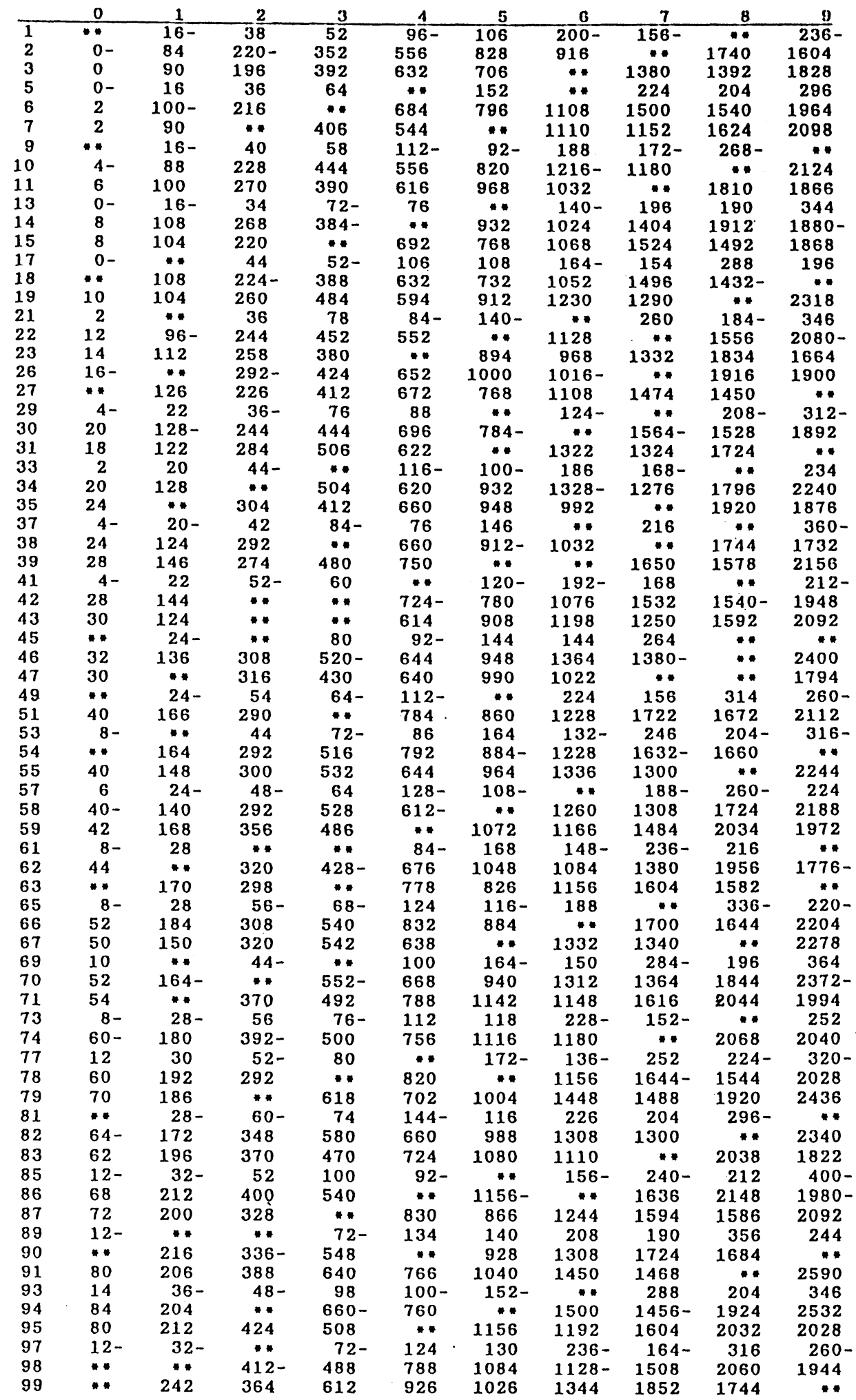


HIROFUMI ISHIKAWA

\begin{tabular}{|c|c|c|c|c|c|c|c|c|c|c|}
\hline & 10 & 11 & 12 & 13 & 14 & 15 & 16 & 17 & 18 & 19 \\
\hline 1 & 356 & 306 & $564-$ & $336-$ & 654 & 476 & $688-$ & $\because$ & $1032-$ & $59 \overline{6}-$ \\
\hline 2 & 2204 & 2656 & 2448 & 3100 & 3976- & 3500 & 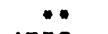 & 5348 & 4500 & 5364 \\
\hline 3 & 2380 & 2282 & 2736 & 3614 & 3128 & 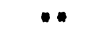 & 4752 & 4296 & 5228 & 5964 \\
\hline 5 & 264 & $468-$ & 308 & 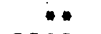 & 428 & 628 & 488 & 916 & $*$ & 976 \\
\hline 6 & 2512 & 2436 & 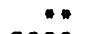 & $3808-$ & 3344 & 4212 & 5020 & 4532- & 5540 & $6808-$ \\
\hline 7 & 1884 & $\bullet$ & 3000 & 2786 & 3540 & 4468 & 3958 & 4576 & 5824 & 4946 \\
\hline 9 & 436- & 272- & 504 & 412 & 592- & 478 & $856-$ & $500-$ & $\because$ & 676 \\
\hline 10 & $2080-$ & 2564 & 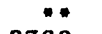 & 3064 & 3548 & 4412 & 3964 & $\because$ & $6072-$ & 5052 \\
\hline 11 & 2308 & 2916 & 2760 & 3476 & 4348 & 3810 & $*$ & 5496 & 4926 & $\bullet$ \\
\hline 13 & $224-$ & 420 & $360-$ & 496- & $\omega$ & 740 & $428-$ & 782 & $*$ & $852-$ \\
\hline 14 & 1 & $3088-$ & 2716 & $\because$ & 4244 & $3852-$ & 4676 & $5576-$ & 5028 & 5868 \\
\hline 15 & 2532 & 2400 & 1 & 3552 & 3388 & 3924 & 4964 & 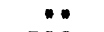 & -1 & 6632 \\
\hline 17 & - & $320-$ & $424-$ & 374 & $656-$ & 396 & $*$ & 588 & 804 & $*$ \\
\hline 18 & $2384-$ & 2216 & 2820 & 3640 & $3244-$ & 3868 & $4896-$ & 4124 & 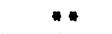 & 6172 \\
\hline 19 & 2150 & 2660 & 3520 & 3010 & 3748 & 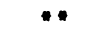 & 4294 & 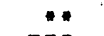 & 6152 & 5568 \\
\hline 21 & 272- & 432 & 340 & 632- & $*$ & 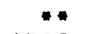 & $572-$ & $772-$ & 618 & $1108-$ \\
\hline 22 & 1948 & 2596 & 3284 & $2860-$ & $*$ & $4368-$ & 3884 & 4604 & 5788 & 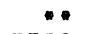 \\
\hline 23 & 2260 & 2738 & 2566 & 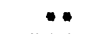 & 4062 & 3590 & 4188 & 5294 & 4598 & 5592 \\
\hline 26 & $\bullet$ & 3000 & $2884-$ & 3364 & 4260 & 3852 & 4852 & 5876 & 4896 & $\because$ \\
\hline 27 & 2420 & 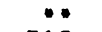 & 2884 & 3698 & 3198 & 4056 & 4658 & 4192 & $\bullet$ & 6376 \\
\hline 29 & $\bullet$ & $512-$ & $316-$ & 586 & $476-$ & 648 & 1 & 956 & 564 & 1034 \\
\hline 30 & 2612 & $2364-$ & 2900 & 3756 & 3468 & $\bullet$ & 4896 & $4648-$ & 5332 & 6420- \\
\hline 31 & 2230 & 2828 & 3402 & $\because$ & 0 & 4738 & 4324 & 5228 & 6406 & 5278 \\
\hline 33 & 424- & 252 & $*$ & 380 & 548- & 448 & 836 & 496- & 856 & $688-$ \\
\hline 34 & 2108 & $*$ & 3536 & 3244 & 3884 & 4936 & 4216 & 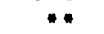 & 6292 & 5688 \\
\hline 35 & $*$ & 3036 & 2676 & 3388 & 4164 & 3804 & 4652 & 5412 & 4992 & $*$ \\
\hline 37 & 244- & 426 & $340-$ & 492 & 414 & 768 & 432- & 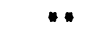 & 612 & $848-$ \\
\hline 38 & 2276 & 2928- & 2556 & 3316 & 3868 & 3604 & $*$ & 5548 & 4784 & 5524 \\
\hline 39 & 2698 & 2436 & 3172 & 4012 & 3658 & $\bullet$ & 5460 & 4644 & 5428 & 6860 \\
\hline 41 & 410 & 340 & 492- & $\cdots$ & 720 & 428 & 794 & $600-$ & 860 & 674 \\
\hline 42 & $2416-$ & 2340 & $\bullet$ & 3608 & 3332 & 4020 & $5060-$ & 4280 & 5372 & 6132 \\
\hline 43 & 2076 & 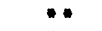 & 3300 & 2960 & 3692 & 4502 & 3888 & 4916 & 5976 & 5128 \\
\hline 45 & 292 & $424-$ & 336 & 648 & 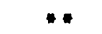 & 696 & 540 & $784-$ & 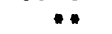 & 1080 \\
\hline 46 & 2188 & 2700 & 3532 & 3260 & 4092 & 4796- & 4320 & 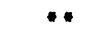 & 6236 & 5532 \\
\hline 47 & 2276 & 2888 & 2740 & 3248 & 3998 & 3620 & * & 5506 & 4670 & 5868 \\
\hline 49 & $380-$ & 318 & $588-$ & 356 & $*$ & 520- & $740-$ & 548 & $\bullet$ & $596-$ \\
\hline 51 & 2766 & 2494 & - & 4044 & 3622 & 4508 & 5340 & 4844 & 5592 & 6854 \\
\hline 53 & $\bullet$ & $484-$ & 300 & 576 & $464-$ & $600-$ & 504 & 896- & $500-$ & 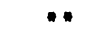 \\
\hline 54 & 2844 & 2540 & 3252 & $3916-$ & 3504 & 4372 & 5556 & 4904- & * & 7068 \\
\hline 55 & 2096 & 2700 & 3504 & 3172 & 3828 & 4836 & 4144 & $*$ & 6140 & 5396 \\
\hline 57 & 436 & 284- & 522 & 388 & 580 & 1 & $816-$ & 476 & 910 & 692 \\
\hline 58 & 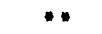 & 2620 & $3300-$ & 3028 & - & 4572 & $3936-$ & 4996 & 5748 & 5028 \\
\hline 59 & 2528 & 3240 & 2882 & $\bullet$ & 4374 & 3950 & 4844 & 6042 & $*$ & 6192 \\
\hline 61 & $256-$ & $\bullet$ & 396- & 552- & 422 & 828 & 492 & 902 & 652- & 960 \\
\hline 62 & $*$ & 2884 & 2760 & 3460 & 3988 & 3756 & 4436 & 5236 & $\bullet$ & $\because$ \\
\hline 63 & 2542 & 2410 & 2924 & 3584 & 3348 & 4264 & 5094 & 4292 & - & 6392 \\
\hline 65 & 420 & $324-$ & 476 & 388 & $736-$ & $428-$ & $*$ & 628- & 816- & 620 \\
\hline 66 & $2828-$ & 2672 & 3180 & 4132 & $3640-$ & 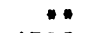 & $\bullet$ & 4832 & 5836 & 6816 \\
\hline 67 & 2092 & 2640 & 3312 & 3074 & $*$ & 4506 & 4146 & 4836 & 5750 & 5268 \\
\hline 69 & $304-$ & 452 & $*$ & $\because$ & 396 & 766 & $556-$ & $816-$ & 632 & 1176 \\
\hline 70 & 2208 & $\bullet$ & 3468 & $3044-$ & $*$ & $4872-$ & 4324 & 4940 & 6268 & $5304-$ \\
\hline 71 & 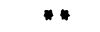 & 3326 & 3052 & 3508 & 4570 & 3938 & 4712 & 5996 & 5454 & $*$ \\
\hline 73 & $368-$ & 320 & 560 & $348-$ & 626 & $*$ & 700 & $*$ & $1036-$ & $568-$ \\
\hline 74 & 2452 & 3180 & 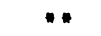 & 3732 & 4592 & 4016 & $\bullet$ & 5872 & 5136 & 6268 \\
\hline 77 & 266 & 512 & $308-$ & $*$ & 452 & 656 & 516 & 916- & 536- & 966 \\
\hline 78 & $*$ & 2460 & $\because$ & $3744-$ & 3528 & 4044 & 4976 & 4468 & 5460 & 6604 \\
\hline 79 & 2396 & $\cdots$ & 3634 & 3404 & 4236 & 5070 & 4356 & 5384 & 6454 & 5562 \\
\hline 81 & 468 & 284- & 552 & $452-$ & 644- & 480 & $\bullet$ & 516- & 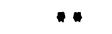 & 732 \\
\hline 82 & $2216-$ & 2708 & 3428 & 3008 & 3660 & 4764 & $-*$ & 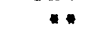 & 5968- & 5276 \\
\hline 83 & $\bullet$ & $*$ & 2838 & 3440 & 4234 & 3886 & 1. & 5506 & 4792 & 5924 \\
\hline 85 & 252 & 484 & $388-$ & 536- & $*$ & $808-$ & $452-$ & 884 & 684 & $960-$ \\
\hline 86 & 2612 & 3268 & 2936 & 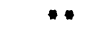 & 4736 & $4144-$ & 4900 & 6180 & 5308 & 6180 \\
\hline 87 & 2742 & 2522 & $*$ & 3948 & 3350 & 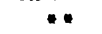 & 5208 & 4634 & 5484 & 6358 \\
\hline 89 & 1 & $340-$ & $516-$ & 390 & $756-$ & 452 & 858 & $640-$ & $888-$ & $\bullet$ \\
\hline 90 & 2736 & 2524 & 3324 & 4128 & $3488-$ & 4484 & 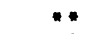 & 4584 & 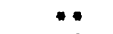 & 6940 \\
\hline 91 & 2314 & 3028 & 3718 & 3344 & 4132 & 5116 & 4632 & $\bullet$ & 6632 & 5632 \\
\hline 93 & $308-$ & $420-$ & 342 & 644 & 392- & -4 & 544- & 792 & 570 & $1088-$ \\
\hline 94 & 2408 & 3020 & 3624 & 3460 & $\bullet$ & 4960- & 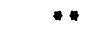 & 5580 & 6696 & $5628-$ \\
\hline 95 & 2468 & 3132 & 2860 & - & 4524 & 3916 & 4908 & 5724 & 5112 & 6092 \\
\hline 97 & $364-$ & $\bullet$ & 596- & 356 & 634 & $520-$ & $688-$ & 550 & 1036 & $588-$ \\
\hline 98 & 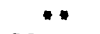 & 3128 & 2728 & 3412 & 4324 & 3932 & 4756 & 5604 & 5012- & $\because$ \\
\hline 99 & 2904 & 2796 & 3484 & 4062 & 3894 & 4556 & 5582 & 4856 & $*$ & 7282 \\
\hline
\end{tabular}


$x \equiv 12 \bmod (16)$, they are nearly the same as for the case of $x \equiv 1 \bmod (8)$. So we omit them. The period of 24 which appears in Fig. 8 are derived by the elliptic part $b(x) h(-3 x)$. Fig. 8 shows that there are not any other periodic factors in $\eta(x)$. We think that the fact above is reflected in the random appearance of elliptic points.

We conclude from these studies that the statistical model is useful to analyze the number theoretical data quantitatively.

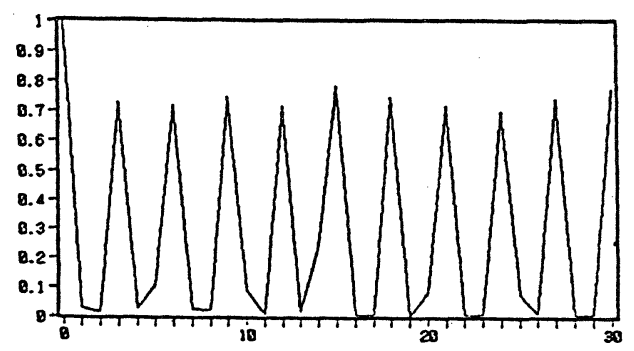

Fig. 7

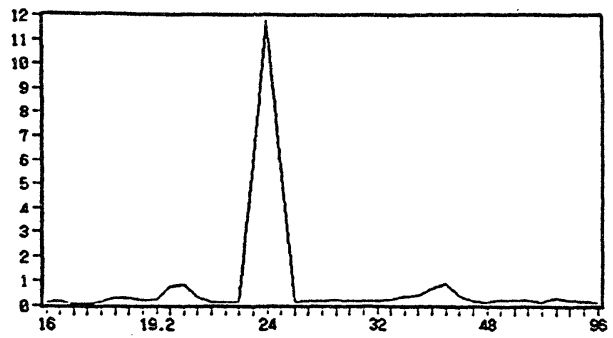

Fig. 8

\section{Table of the dimensions of $S_{2}(\Gamma)$}

For a square-free number $D(1<D<2000)$, we tabulate the dimensions of $S_{2}(\Gamma)$ over real quadratic fields $\mathbf{Q}(\sqrt{D})$. The number $D$ is given by

$$
D=i+100 j(i=\text { row number, } j=\text { column number })
$$

When the mark '-' appears after a figure, $\mathbf{Q}(\sqrt{D})$ has a unit of negative norm. In this case, the dimension of the space of cusp forms on upper half space of $\mathbf{C}^{2}$ is equal to the quarter of the dimension in the table. The mark ' $* *$ ' means $D$ is not square-free. For the case of $D=2,3,5,13,17, \operatorname{dim} S_{2}(\Gamma)$ vanishes exactly.

\section{REFERENCES}

Hirzebruch, F. E. P. (1973). Hilbert modular surfaces, L'Enseignement Math., 19, 183-281. Ishikawa, H. (1979). The traces of Hecke operators in the space of the 'Hilbert Modular' type cusp forms of weight two, Scientific Papers C. General Education Univ. Tokyo, $29,1-28$.

Prestel, A. (1968). Die elliptischen Fixpunkte der Hilbertschen Modul-gruppen, Math. Ann., 177, 181-209.

Shimizu, H. (1963). On discontinuous groups operating on the product of the upper half planes, Ann. of Math., 77, 33-71. 


\section{HIROFUMI ISHIKAWA}

Siegel, C. L. (1969). Berechnung von Zeta funktionen an ganzzahligen Stellen, Nachr. Akad. Wiss. Gottingen, 10, 87-102.

(Received April 1988; Revised May 1988.) 